Gefässchirurgie 2021 $26: 347-358$ https://doi.org/10.1007/s00772-021-00802-z Angenommen: 29. Juni 2021 Online publiziert: 13. August 2021 ๑) Springer Medizin Verlag GmbH, ein Teil von Springer Nature 2021

\section{Innovationen in der endovaskulären Therapie der peripheren arteriellen Verschlusskrankheit (pAVK)}

\author{
Jörg Teßarek' · Alexander Oberhuber ${ }^{2}$ \\ ' Klinik für Gefäßchirurgie, Bonifatius Hospital Lingen, Lingen, Deutschland \\ ${ }^{2}$ Klinik für Vaskuläre und Endovaskuläre Chirurgie, Universitätsklinikum Münster, Münster, Deutschland
}

\section{Zusammenfassung}

\section{In diesem Beitrag}

- Methode

- Neuerungen in der Therapie der peripheren arteriellen Verschlusskrankheit (pAVK)

Intraoperative Bildgebung und Strahlenreduktion - Neuerungen im Bereich der Carrier-Systeme • Drahttechnik • Re-Entry-Systeme $\cdot$ Neuerungen im Bereich der "therapeutischen" Systeme • Atherektomiesysteme

- Diskussion

Die Kooperation von Medizinern, Ingenieuren und anderen Naturwissenschaften bringt weiterhin effektive Produkte auf den Markt, die dazu geeignet sind, die Dominanz der endovaskulären Techniken im Bereich der peripheren arteriellen Verschlusskrankheit ( $p A V K$ ) weiter auszubauen. Begleitend werden zunehmend bildgebende Verfahren weiterentwickelt oder neu entwickelt, die helfen können, die Strahlenbelastung für Patient und Anwender trotz wachsender Zahlen strahlenbasierter Therapieverfahren deutlich zu reduzieren. Mittlerweile haben wir ein Materialportfolio an der Hand, das es erlaubt, jede Gefäßregion endovaskulär zu behandeln. Auf der anderen Seite dürfte das endovaskuläre Leistungsgefüge nicht flächendeckend homogen gestaltet sein und die jeweilige Ergebnisqualität dann für die Chirurgie sprechen. In diesem Übersichtsartikel sollen neue Materialien und neuere Ergebnisse vorgestellt werden, die den Bereich der endovaskulären Behandlung der pAVK in allen Gefäßsegmenten mit beeinflussen

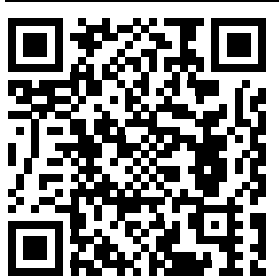

QR-Code scannen \& Beitrag online lesen dürften.

\title{
Schlüsselwörter
}

Atherektomie $\cdot$ Kritische Extremitätenischämie $\cdot$ Stenose $\cdot$ Katheter $\cdot$ Rekanalisierung

\section{Einleitung}

Kaum ein Bereich der Gefäßtherapie hat sich in den vergangenen Dekaden derartig weiterentwickelt wie der der endovaskulären Methoden und Materialien. Mit der Publikation der BASIL-Studienergebnisse in 2005 [1] und 2010 [2], die eine Gleichwertigkeit der schlichten femoropoplitealen Ballon-PTA (perkutane transluminale Angioplastie) mit dem Bypassverfahren beim Vorliegen einer kritischen Extremitätenischämie nachweisen konnte, schien die konventionelle Gefäßchirurgie in der bisherigen Dynamik der fachlich kontroversen Diskussion einzuhalten. Die 2019 im JVS veröffentlichen englischen Daten über das Outcome femoropoplitealer Bypässe bei Patienten mit kritischer Extremitätenischämie zwischen 2009 und 2014 zeigten zwar weniger perioperative Komplikationen, aber dafür eine statistisch signifikante
Verschlechterung der Bypassergebnisse im Vergleich zur BASIL-Studie [3].

I) No-go-Regionen für das endovaskuläre Vorgehen scheint es nicht mehr zu geben

Dem steht, nur als Beispiel für den andauernden technischen Fortschritt im endovaskulären Sektor, die wachsende Zahl an Publikationen über Atherektomie mit und ohne den Einsatz von Medikamenten beschichteten Ballons [4] bei schwer kalzifizierten komplexen femoropoplitealen Läsionen gegenüber, die die Bypasschirurgie als primäre Option bei diesen Läsionen in Frage stellt. No-go-Regionen für das endovaskuläre Vorgehen scheint es nicht mehr zu geben. Sowohl die A poplitea wie auch die A. femoralis communis sind lange keine unangefochtenen Bastionen der offenen Chirurgie mehr $[5,6]$. 
Die mediale Präsenz der endovaskulären Techniken mit technischen Erfolgsraten von $95-96 \%$ für die Rekanalisierung kalzifizierter TASC-IIC- oder -D-Läsionen (TransAtlantic Inter-Society Consensus) durch z. B. die Atherektomie [7, 8] ist erdrückend, auch wenn unterstellt werden kann, dass viele Studien und die dazugehörigen Publikationen von der Industrie gesponsort oder getriggert wurden.

\section{Methode}

Auch wenn Corona-bedingt die 14 Monate kaum Möglichkeiten der Rekrutierung von Studienpatienten mit einer geregelten Nachuntersuchung zuließen, so wurden in diesem Zeitraum von 2019 bis heute interessante Studienergebnisse publiziert, die sich auf mittlerweile in der Fläche eingesetzte Entwicklungen der letzten 3 Jahre beziehen. Auch Innovationen, deren klinische Wertigkeit bisher nicht durch publizierte Studienergebnisse gestützt wird, werden aufgeführt, da aktuell wieder Studien anlaufen und diese Systeme in naher Zukunft von Interesse sein werden. Relevante Publikationen wurden recherchiert und analysiert.

Die Auflistung gliedert sich in den Bereich der intraoperativen Bildgebung und Strahlenreduktion, der Carrier-Systeme, wie Drähte und Schleusen und das Segment der therapeutisch aktiven Systeme, wie Ballons, Stents und Atherektomiesysteme. Es besteht nicht der Anspruch auf Vollständigkeit, die aufgeführten Systeme sprechen aber den weniger wie den sehr erfahrenen endovaskulär tätigen Gefäßchirurgen an und reflektieren die Bandbreite der Neuerungen in gleicher Weise wie den Trend hin zur endovaskulären Versorgung auch sehr komplexer Läsionen.

\section{Neuerungen in der Therapie der peripheren arteriellen Verschlusskrankheit (pAVK)}

\section{Intraoperative Bildgebung und Strahlenreduktion}

Endovaskuläre Verfahren sind Röntgen-basierte Untersuchungen und gehen mit einer Strahlenbelastung für Patienten und Anwender (OP-Team inklusive Anästhesie) einher. Mit der wachsenden Komplexität der Prozeduren und der wachsenden Zahl Röntgen-basierter Eingriffe gewinnt der Strahlenschutz primär durch Vermeidung von Röntgenstrahlen eine herausragende Wichtigkeit.

Das FORS ${ }^{\circledR}$-System (Fiber Optic Reality Shaped, Philips bv., Eindhoven, Niederlande; [9]) nutzt nachgewiesener Maßen erfolgreich die Kombination von Image-Fusion-Technik, Hardware als Teil der Röntgenanlage und fiberoptisch ausgerüsteten Kathetern und Drähten, um einzelne Schritte der geplanten Intervention gänzlich ohne Durchleuchtung durchzuführen. Dazu wird das zu behandelnde Gefäßsegment als virtuelles Bild mit allen Bewegungsmöglichkeiten in den Datensatz der Röntgenanlage integriert. Systeme, deren Physiologie und Materialeigenschaften als Datensatz hinterlegt sind, können mittels virtueller Realität dargestellt und kontrolliert bewegt werden. Röntgenstrahlen sind nur noch dann notwendig, wenn nicht erfasste Systeme zum Einsatz gelangen oder eine Angiographie notwendig ist. In diese Richtung geht auch der Einsatz von Kathetersystemen (Track cath ${ }^{\circledR}$, Medyria AG, Winterthur, Schweiz), die über hochempfindliche Sensoren die Flussabweichungen an Gefäßabgängen messen und damit eine nicht Strahlen basierte Sondierung ermöglichen sollen [10] Es wäre durch weitere Studien zu klären, welchen Stellenwert diese Technik in der pAVK-Versorgung einnehmen können wird.

Eine weitere wichtige Technik, die als Ersatz für hochenergetische Strahlenanwendungen (also Angiographien) genutzt werden kann, ist der intravaskuläre Ultraschall. In der eigenen Anwendung konnte dadurch eine Reduktion der Strahlenbelastung bei T/EVAR („thoracic endovascular aortic repair") um $96 \%$ ohne Einfluss auf die prozedurale Sicherheit erreicht werden [11].

Zudem zeigten sich eine höhere diagnostische Sicherheit in Bezug auf die Größe und Beweglichkeit intimaler Lefzen [12] und bessere Ergebnisse hinsichtlich des aortalen Remodelling nach IVUS-gesteuerter (intravaskulärer Ultraschall) Therapie $[13,14]$.

Die Überlegenheit des IVUS gegenüber der kontrastmittelgestützten Bildgebung im Rahmen der endovaskulären Therapie von iliakalen Venenstenosen ist durch die in den vergangenen 3 Jahren veröffentlichten Studienergebnisse noch deutlicher herausgearbeitet worden $[15,16]$. Dabei ist der Aspekt der Strahlenreduktion aber nur wenig beachtet worden. Gerade im Körperstammbereich gewinnt dieser Vorteil des IVUS an Bedeutung, da durch das Mehr an biologischer Masse und die Schrägprojektionen die Strahlenbelastung enorm anwächst. So konnten Montminy et al. [16] nachweisen, dass die Phlebographie in $66 \%$ der Fälle die maximale Stenosezone und den maximalen Stenosegrad nicht korrekt darstellte. Bereits 2017 hatten Gagne et al. [17] in einer prospektiven multizentrischen Studie zeigen können, dass IVUS in $26,3 \%$ der Fälle Stenosen detektieren konnte, die die Phlebographie nicht gezeigt hatte. In einer Subgruppenanalyse konnte die gleiche Arbeitsgruppe auch den prädiktiven Wert der IVUS Messungen hinsichtlich des klinischen Outcomes nachweisen, der der Phlebographie gänzlich fehlte [18]. Die Wertigkeit des IVUS für die endovaskuläre Therapie der aortoiliakalen Gefäßsegmente hatten Buckley et al. [19] bereits 2002 beschrieben. In der damaligen Studie wiesen die angiographisch gesteuerten Interventionen eine 12-Monats-Restenoserate von > 18\% gegenüber $0 \%$ für IVUS auf.

\section{॥ Die Wertigkeit des IVUS für die endovaskuläre Therapie der aortoiliakalen Gefäßsegmente ist hoch}

Die 2020 publizierte Metaanalyse von Sheik et al. [20] mit 8 Studien und 93.551 Patienten und einem durchschnittlichen Follow-up von 24,2 \pm 15 Monaten konnte nachweisen, dass die Sofort- und Langzeitergebnisse für IVUS- und angiographiegesteuerte Interventionen gleich waren, die Rate an perioperativen „adverse events" signifikant $(p=0,006)$ geringer und die der vaskulärer Komplikationen deutlich geringer war als bei den angiographisch gesteuerten Maßnahmen $(p=0,013)$.

Neben der Reduktion von Strahlung führt der Einsatz des IVUS auch zur Verbesserung der Langzeitergebnisse und reduziert die perioperativen Komplikationsraten. Im koronaren Segment zeigt sich 


\section{STANDARD \\ INTRODUCER \\ SHEATH}

GLIDESHEATH

SLENDER

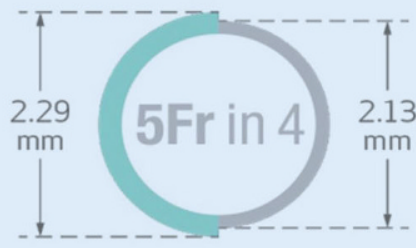

STANDARD

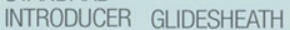

SHEATH

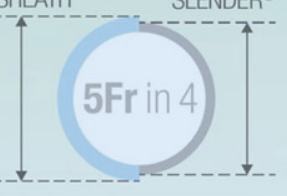

Inner

Diameter

$5 \mathrm{Fr}$
STANDARD

INTRODUCER GLIDESHEATH

SHEATH SLENDER
STANDARD

INTRODUCER GLIDESHEATH

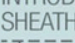
SLENDER

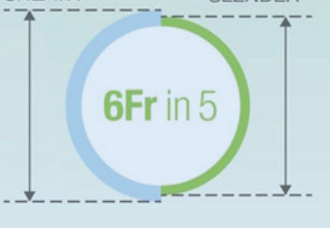

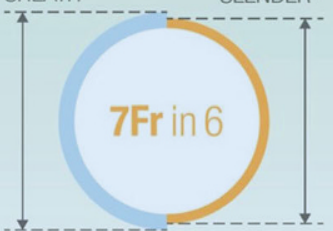

$7 \mathrm{Fr}$

Abb. $1 \Delta$ Die Abbildung verdeutlicht die Reduktion des Außendurchmessers der Glide Sheath Slender ${ }^{\circledR}$ im Vergleich zu einer Standardschleuse. Mit der Verringerung des Außendurchmessers für die 5-bis 7-F-Schleuse gehen auch eine Reduktion des Punktions- und des Wandtraumas über die gesamte Kontaktlänge von Schleuse und Gefäßintima einher. Gerade die langstreckige Dissektion kleinlumiger Zugangsgefäße am Arm oder Unterschenkel sollte dadurch weniger wahrscheinlich werden.

(Mit freundl. Genehmigung, Images used with permission of Terumo Europe NV, $\odot ~ 2020$, all rights reserved)

durch den Einsatz des IVUS als bildgebende Technik sogar eine statistisch signifikante Reduktion des kardiovaskulären Todes, des Herzinfarktes sowie der Revaskularisationsrate und der Stentthrombose [21].

Aktuell wird eine Studie zur IVUS-gesteuerten Evaluation der Bridging-Stents statt eines Cone-beam-CT im Rahmen von bEVAR durchgeführt [22]. Diese Vorgehensweise dürfte dann ohne die für die Röntgen-basierte Evaluation notwendige Strahlenbelastung auskommen. In der Gefäßperipherie und der koronaren Stentversorgung hat sich der IVUS ja schon als valides Bildgebungsverfahren bewiesen.

\section{Neuerungen im Bereich der Carrier- Systeme}

\section{Schleusentechnik}

Auch wenn die Ausweitung der therapeutisch und diagnostisch genutzten Gefäßzugänge für die Koronarintervention und mittlerweile auch die pAVK hin zum Radialiszugang kontrovers diskutiert werden kann [23-25], so hat die Industrie auf die zugangsbedingten Komplikationen reagiert und extra dünnwandige Schleusen entwickelt, die bei gleichem Arbeitslumen einen um $1 \mathrm{~F}$ verringerten Außendurchmesser aufweisen. Zu dieser Gruppe gehört die von Terumo hergestellte Glide Sheath Slender ${ }^{\circledR}$ (Terumo Interventional Systems, Tokio, Japan), die in den Größen $5 \mathrm{~F}, 6 \mathrm{~F}$ und $7 \mathrm{~F}$ mit einer Schaftlänge von 10 und $16 \mathrm{~cm}$ angeboten wird. Die $\mathbf{0}$ Abb. 1 zeigt die Unterschiede zwischen Standard- schleuse und dem dünnwandigen Pendant auf.

Die so abgewandelten Schleusen können in gleicher Weise zur Sicherung des arteriellen Zugangs auch über vulnerable Unterschenkelgefäße mit kleinem Durchmesser eingesetzt werden. Erstaunlicherweise sind bisher lediglich Publikationen aus dem kardiologischen Bereich (also in Bezug auf den tranradialen Zugang) bekannt, nicht aber aus dem peripheren Segment. Gerade für die immer häufiger genutzten retrograden Zugänge über die Unterschenkelgefäße dürfte sich hier ein wesentlicher Vorteil nachweisen lassen, da damit auch eine von distal durchführbare angiographische Kontrolle der Interventionsschritte möglich ist [26].

Mit der Weiterentwicklung langer Schleusen und Katheter-/Ballonschäfte wird die transradiale Intervention als Alternative sicherlich weiter propagiert werden. Die kruralen oder pedalen Zugänge sind mittlerweile Routine [27], sie sind aber nur dann nutzbar, wenn diese Gefäße noch durchgängig sind. Gerade beim Diabetiker findet sich häufig die Kombination aus schwer kalzifizierten Läsionen und einer Rarefizierung der distalen oder akralen Gefäßsegmente.

\section{Steuerbare Schleusen- und}

\section{Führungskatheterysteme}

Die Steuerungstechnik für Schleusen und Führungskatheter ist zwar nicht neu, aber es zeigt sich zunehmend die Nutzbarkeit dieser Systeme bei der Behandlung bei arteriellen und venösen Verschlussszenarien und nicht nur bei der Kanülierung komplexer Aortenendoprothesen. Neben der Oscor Destination Twist ${ }^{\circledR}$ (Oscor Inc., Abbott Park, IL, USA, 6,5-13,8F, 0-190 ) als unidirektional steuerbare Schleuse und der Destino ${ }^{\circledR}$ und Destino reach $^{\circledR}(8,5-12 \mathrm{~F}$, $0-180$ ) als bidirektional Schleuse (Oscor Inc.) mit den Arbeitslängen von 45, 55, 65 und $90 \mathrm{~cm}$ wird auch von Abbot eine bidirektional steuerbare Schleuse angeboten,

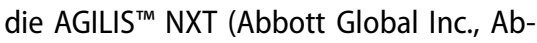
bott Park, IL, USA). Die Fustar ${ }^{\circledR}$ (LaMed, Oberhaching, Deutschland) ist eine weitere unidirektional im Spitzenbereich von $0-160^{\circ}$ beugbare Schleuse und ist in den Größen von 5-10 F mit Längen von 55, 70 und $90 \mathrm{~cm}$ erhältlich.

॥) Die Vorteile der steuerbaren Schleusentechnik liegen im direkten Drahtzugang zu den Zielgefäßen

Die Vorteile dieser Schleusentechnik liegen im direkten Drahtzugang zu den Zielgefäßen, z.B. der A. mesenterica superior (- Abb. 2) oder einem Prothesenschenkelverschluss (- Abb. 3), ohne dabei Führungskatheter einsetzen zu müssen, da sie viele Katheterkonfigurationen imitieren können. Dies bietet aber u.a. die Option, die Schleusenspitze und den Schaft in einer stabilen Position in einem Gefäßstumpf zu fixieren und dadurch die Penetrationskraft und den gezielten Vorschub eines Drahtes zu unterstützen (ब Abb. 3). Die Rekanalisierung eines verschlossenen Zielgefäßes, sei es arteriell oder venös, kann dadurch wesentlich vereinfacht oder erst 


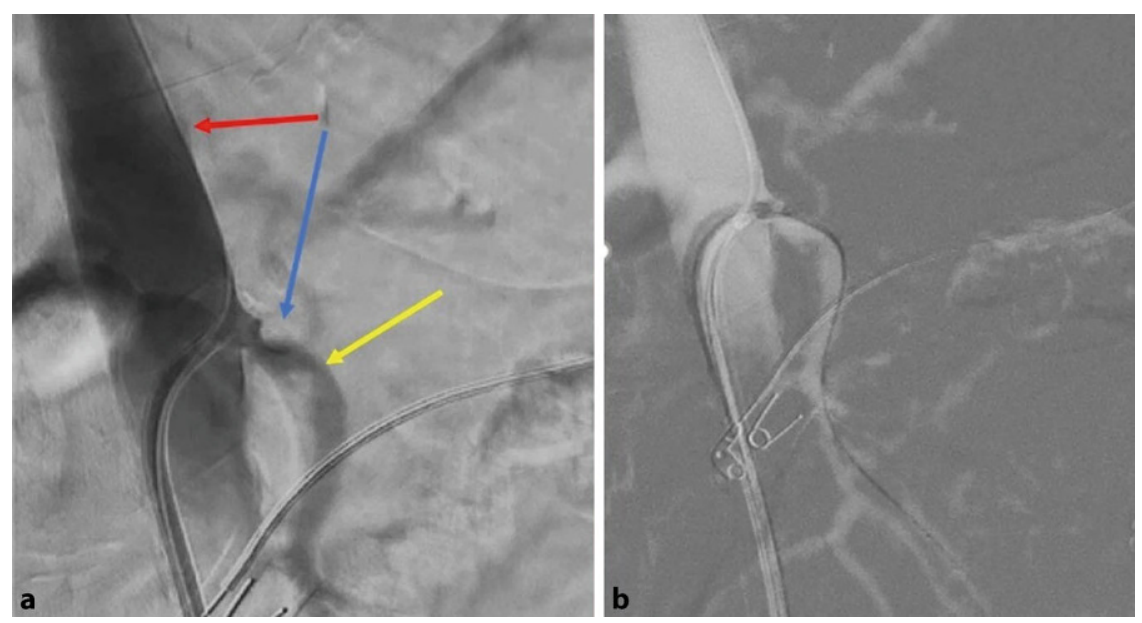

Abb. $2 \Delta$ a Hochgradige, irregulär konfigurierte Tandemstenose (blauerund gelber Pfeil) der A. mesenterica superior (AMS) bei Verschluss des Truncus coeliacus (roter Pfeil). b Die teilflektierte OscorSchleuse erlaubte die direkte Drahtsondierung mittels 14er-Hybriddraht und die Ballonierung mit anschließender Stentversorgung. (Mit freundl. Genehmigung J. Teßarek, Bonifatius Hospital Lingen)

ermöglicht werden, da die Schleuse deutlich mehr Formstabilität aufweist als ein Katheter und damit der Kraftvektor eines Drahtes stabil ausgerichtet werden kann.

Auf die Entwicklung steuerbarer Mikrokatheter wurde im Oktober 2020 mit der Bekanntgabe der Food and Drug Administration (FDA) und CE-Zertifizierung für den 2,1-F-BENDIT ${ }^{\circledR}$ Neuro-Mikrokatheter (Bendittech Inc., Petach Tikva, Israel) aufmerksam gemacht. Mittlerweile wird auch ein 2,7-F-Mikrokatheter für periphere Interventionen angeboten. Die supraselektive Sondierung komplexer Gefäßanatomien sollte dadurch deutlich erleichtert und weniger zeitaufwendig werden. Der von Merit Medical angebotene und seit 2018 erhältliche Mikrokatheter SwiftNINJA ${ }^{\circledR}$ (Merit Medical Inc., South Jordan, UT, USA) ist ebenso ein steuerbarer 2,7 F Katheter, der über einen 18eroder kleineren Draht laufen kann. Bisher liegen allerdings nur Erfahrungen aus der Kardiologie [28] und von Embolisationen vor [29].

Nicht nur im neuroradiologischen und kardiologischen Gefäßsegment oder bei komplexen Organembolisationen dürften solche Techniken von Vorteil sein, da sie bei höherer Erfolgsrate die Prozedur- und Durchleuchtungszeit und damit Patientenund Anwenderbelastung verringern. Inwieweit die Systeme beim Einsatz in der Gefäßperipherie bezüglich der Kostenerstattung abbildbar sein werden, wird abzuwarten sein. Eine ökonomische Betrach- tung von höherpreisigen Schleusen- oder Kathetersystemen müsste dann auch die OP-Zeit und die Kosten für frustrane oder mit Komplikationen behaftete Prozeduren mit einbeziehen. Aus Sicht der Anwender sollte der Einsatz jeglichen Materials, das die Strahlenbelastung von Patient und OPTeam zu reduzieren hilft auf jeden Fall in Betracht gezogen werden.

\section{Drahttechnik}

Führungsdrähte haben die Aufgabe, die Läsion oder ein Gefäß zu passieren, das endovaskuläre Instrument an den Ort des Geschehens zu bringen und über den gesamten Zeitraum der Prozedur den Zugang zur Läsion oder dem Zielgefäß zu erhalten. Damit sind sie ein wichtiger, manchmal sogar der wichtigste Bestandteil einer Gefäßintervention. Je nach Komplexität der Prozedur können mehrere Drähte für den Transport oder die Passage einer Zielläsion notwendig sein, was sowohl die Materialkosten hebt, aber auch mehr Manipulation, Risiko und Strahlenbelastung für alle Beteiligten bedeutet. Die Neuerungen in diesem Bereich zielen darauf ab, sog. „Allrounderdrähte" zu entwickeln, um Drahtwechsel redundant werden zu lassen und das Prozedurrisiko zu senken.

Bei der Weiterentwicklung eines Drahtes spielt die Kombination verschiedener Werkstoffe die entscheidende Rolle. So können Drahtspitze, Schaft und Beschichtung durch die Veränderung der Längen- verhältnisse, der Härtegrade der Werkstoffe und die Ausrichtung der Polymerbeschichtung in Form von longitudinal oder zirkulär ausgerichteten Faserbündeln die Eigenschaften des Drahtes oder einzelner Drahtsegmente beeinflussen. Dass nicht nur die Drahtdicke die Stabilität beeinflusst zeigt die $\mathbf{0}$ Abb. 4.

Der 0,0018"-Glidewire Advantage ${ }^{\circledR}$ (Terumo Medical Systems Inc., Tokio, Japan) hat im Testverfahren in der Kombination mit dem jeweils gleichen Katheter eine höhere Biegeresistenzals ein 0,0035" Stiff-Draht des gleichen Herstellers. Ziel dieser Modifikationen am Drahtmaterial ist die Befähigung dünnerer Drähte zur Penetration einer stark kalzifizierten Läsion, um dann einen Ballon mit kleinerem Passageprofil nachfolgen zu lassen. Das Passageprofil eines 0,0035"'-gängigen 3-mm-Ballons kann bei $4,8 \mathrm{~F}$ liegen, dass eines 0,0018"- oder 0,0014"-gängigen Ballons bei 3,5 oder 2,9F.

Zudem erlaubt die kurze Spitze $(1 \mathrm{~cm})$ in Kombination mit dem harten Drahtschaft ein sehr gutes haptisches Feedback für den Anwender. Die Kombinationen von Drahtmodifikation und Reduktion des Passageprofils für Supportkatheter oder BalIons repräsentiert eine Möglichkeit zur Behandlung komplizierter Läsionen mit dem einfachsten Standardverfahren, der POBA („plain old balloon angioplasty“). Mittlerweile haben die meisten Anbieter derartige Drähte im Portfolio, um das eigene Materialportfolio mit anbieten zu können. Dies dürfte dann auf Kompatibilität getestet sein.

\section{I) Die individuell geltenden Limitationen für Führungsdrähte müssen unbedingt beachtet werden}

Eine interessante Neuerung repräsentiert der Marsman Speedwire ${ }^{\circledR}$ (Mermaid Medical, Hilversum, Niederlande). Dieser Draht ist speziell für den antegraden Zugang über die A. femoralis communis und A. femoralis superficialis entwickelt worden, um die unerwünschten Sondierungen der A. profunda femoris zu vermeiden helfen. Der 0,0035"-Draht hat eine besondere Formgebung mit J-Spitze und langbogigem Schaft bei einer Gesamtlänge von $70 \mathrm{~cm}$. Dadurch soll er sich beim Einführen in die Schleuse selbstführend zur ventra- 


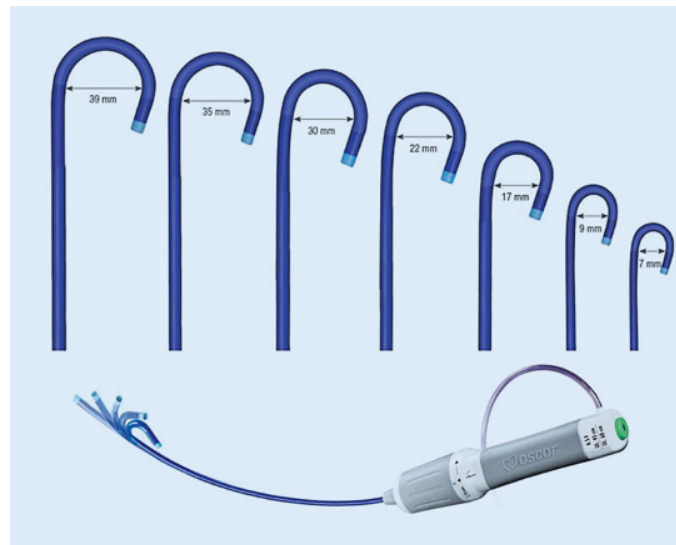

a
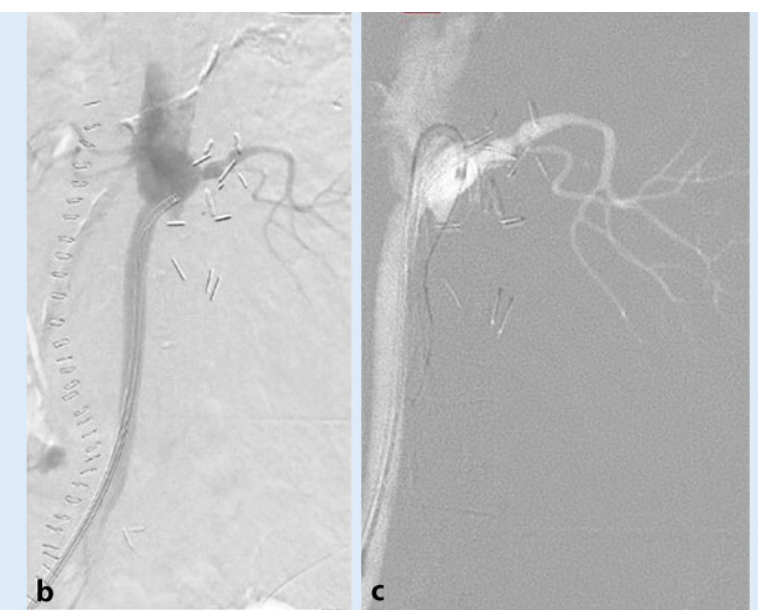

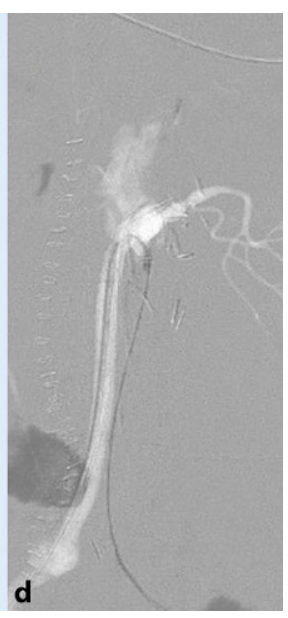

Abb. $3 \Delta$ a Steuerbare Schleuse mit 0-190 Beugefähigkeit (7,5 F Oscor Destino Twist ${ }^{\circledR}$ ) im Anastomosenbereich einer suprarenal angeschlossenen Y-Prothese. Am OP-Tag kam es zum Verschluss des linken Prothesenschenkels. $\mathbf{b}$ Die Rekanalisierung konnte ohne Manipulation an der transaortal desobliterierten und reinserierten Nierenarterie und der Anastomose erfolgen. c Die Destino-Twist-Schleuse wurde dazu oberhalb der Anastomose um $180^{\circ}$ flektiert und in dieser Stellung in den Thrombus gezogen (d), um durch den Support eine direkte Drahtpassage zu unterstützen und gleichzeitig Thrombusverwirbelungen mit der Gefahr der Embolisation in die benachbarte Nierenarterie zu verhindern. (a Mit freundl. Genehmigung Oscor Inc., USA. b, c Mit freundl. Genehmigung J. Teßarek, Bonifatius Hospital, Lingen, Deutschland)

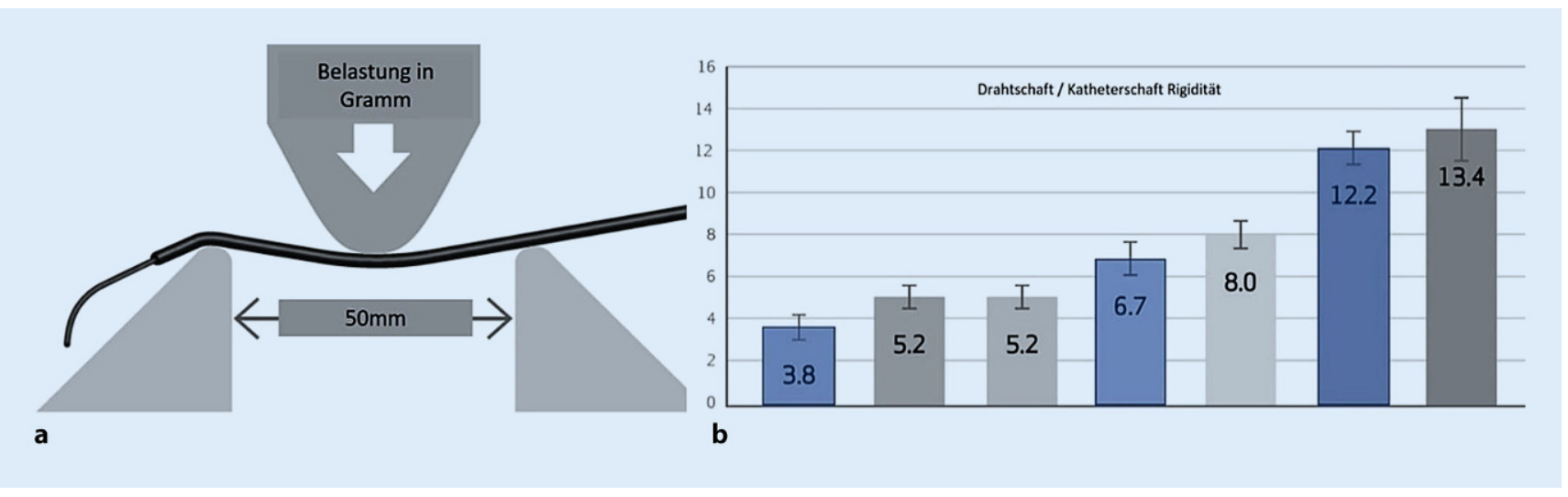

Abb. 4 \ Benchmark-Tests für die Biegesteifigkeit von Draht-/Katheterkombinationen in einem standardisierten Versuchsaufbau (a). Die blau hinterlegten Säulen im Diagramm (b) geben die Grammzahl für die definierte Biegung wieder. Ganz links ist der 35er-Glidewire mit 3-cm-Spitze, in der Mitte der 35er-Glidewire StiffShaft mit 3-cm-Spitze (Mitte) und ganz rechts der 18er-Glidewire Advantage mit 1-cm-Spitze abgebildet. (Mit freundl. Genehmigung, Images used with permission of Terumo Europe NV, @ 2020, all rights reserved)

len Wand des Gefäßes und damit weg vom Profundaostium bewegen.

Trotz aller Verbesserungen der Drahttechnik müssen die individuell geltenden Limitationen für den jeweiligen Draht unbedingt beachtet werden, um Komplikationen zu vermeiden. Die in den Anwenderbeschreibungen („instruction for use“, IFU) aufgeführten "no go's" schließen das Einführen eines polymerbeschichteten Drahtes über eine angeschliffene Stahlkanüle mit der Gefahr der Materialabscherung und v.a. das Rotieren des Drahtes im Verschlusssegment in nur eine Richtung (s. auch IFU Glidewire
Advantage ${ }^{\circledR}$, Terumo Medical Systems, Tokio, Japan; Command ${ }^{\circledR}$ Draht, Abbott Global Inc., Abbott Park, IL, USA usw.) mit ein. Diese Überbeanspruchung der Dehnbarkeit von Drahtkern und Beschichtung kann zur Schädigung des Drahtes und zum Verbleib von Drahtanteilen im Körper führen.

\section{Re-Entry-Systeme}

Die Rekanalisierung von Gefäßverschlüssen kann ante- oder retrograd intraluminal oder subintimal durchgeführt werden. Der wesentliche Faktor für den Erfolg einer auf die Wiederherstellung des Blutflusses ausgelegten Intervention, unabhängig vom betroffenen Gefäßsegment, ist die Passage eines Drahtes oder mehrerer Drähte und deren Platzierung im Gefäßlumen distal des Verschlusses.

Eine seit langer Zeit genutzte Vorgehensweise ist die subintimale Passage mit Rückführung des Drahtes in den intraluminalen Raum durch ein Re-Entry-System [30]. Dies geht in der aortoiliakalen wie femoropoplitealen Strecke aber nur bei Nativgefäßen ohne liegende Stentimplantate. In dem Fall muss intraluminal reka- 


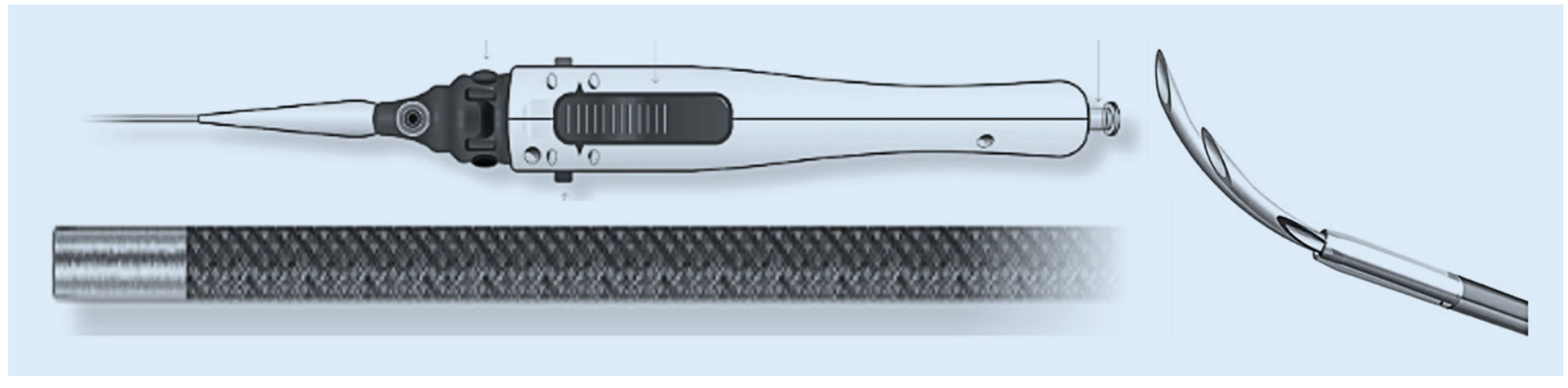

Abb. $5 \Delta$ Go Back ${ }^{\circledR}$-Katheter: Erwird bei eingefahrener Nadel über einen 14er oder 18ernicht hydrophil beschichteten Draht vorgeschobenen. DieBiegung der Nadel erlaubt das Re-Entry bei subintimaler Passage aber auch die scharfe Penetration einer fibrotischen oder kalzifizierten Verschlusskappe, um den Drahtvorschub in die Verschlussstrecke gesteuert zu ermöglichen. (Mit freundl. Genehmigung upstream perpheral technologies Ltd.)

nalisiert werden, um schwerwiegende Gefäßverletzungen zu vermeiden.

Das bekannteste dieser Systeme dürfte das OUTBACK ${ }^{\circledR}$-System der Firma Cardinal Health (Cardinal Health Deutschland, Norderstedt, Deutschland) sein, das als Elite ${ }^{\circledR}$ oder LTD ${ }^{\circledR}$-System angeboten wird. Dessen Nutzung wird mittlerweile auch bei der subintimalen Passage in den distalen Popliteasegmenten und den proximalen Unterschenkelgefäßen beschrieben [31], genauso wie die Nutzung bei distalem retrograden Zugang über ein Unterschenkelgefäß [32].

In der eigenen Klinik wurde das System bisher in der überwiegenden Zahl der Fälle für die subintimale Rekanalisierung aortoiliakaler Verschlüsse eingesetzt, um aortal oberhalb des Verschlusses die Drähte sicher in das Lumen vorzubringen.

Alternativ kann ein IVUS-gesteuertes Re-Entry-System (Pioneer Plus ${ }^{\circledR}$, Philips, Amsterdam, Niederlande), das bereits 2016 auf den Markt gekommen ist oder ein neues System, der GoBack ${ }^{\circledR}$ Crossing \& Re-entry-Katheter (Upstream Peripheral Technologies Ltd., Aran, Israel), zur Anwendung gelangen.

Der GoBack ${ }^{\circledR}$-Katheter ist seit 2020 in Längen von 80 oder $120 \mathrm{~cm}$ lieferbar und kann mit einem Passageprofil von 2,9 oder $4 \mathrm{~F}$ geordert werden. Er läuft über 14erund 18er-Drähte. Für den antegraden Zugang kann eine 5-F-Zugang genutzt werden, für den Crossover-Zugang muss eine 6-F-Schleuse eingebracht werden. Das Prinzip mit Vorschub einer gebogenen und spitz zugeschliffenen Hohlnadel (• Abb. 5) zum Re-Entry erlaubt zudem den Einsatz als Penetrationshilfe, um den Draht durch eine harte Verschlusskappe zu transportieren.

Neuerungen im Bereich der "therapeutischen" Systeme

\section{Ballons}

Neuerungen im Segment der Dilatationsballons waren in den vergangenen Jahren darauf ausgelegt, die dilatative Wirkung der Inflation zu erhöhen und das primär erreichte Lumen zu stabilisieren, um die Notwendigkeit eines Stentimplatats zu verringern. Bereits die prolongierte Dilatation konnte auch bei langen Läsionen des femoropolitealen Gefäßsegments gute Erfolge erzielen [33]. Fibrotische Läsionen oder starke kalzifizierte exzentrische oder/und zirkumferente Plaques können aber weiterhin als Herausforderung für die endovaskulären Verfahren angesehen werden. Die resistenten Wandanteile können ein Recoil zeigen oder mit ausgedehnten Dissektionen auf das Barotrauma reagieren. Daher wurden verschiedene Veränderungen der Ballonkörper vorgenommen, um die Effektivität der Dilatation zu erhöhen und gleichzeitig flussrelevante Dissektionen zu vermeiden, z. B.in die Ballonoberfläche eingelassene gezahnte Leisten, wie dies beim 2017 vorgestellten Serranator $^{\circledR}$ Ballon (Cagent Vascular, Wayne, PA, USA) der Fall ist. Die Ergebnisse der PRELUDEStudie wurden 2019 veröffentlicht [34].

Eine Neuentwicklung in diesem Bereich, die 2020 eine FDA-Zulassung bekommen hat, stellt das XO score ${ }^{\circledR}$-System (Transcient Scientific, Salt Lake City, UT, USA, https://www.xoscore.com/xo-score) zur Gefäßwandpräparation dar. Dabei handelt es sich nicht um einen Ballon, sondern um einen Katheter mit einem Nitinolgitterwerk an der Spitze, das sich nach Einführen eines Ballons als Exoskelett um den aufgeblasenen Ballonkörper legt, so dass ähnlich einem Scoring-Ballon die tangierten Gefäßwandanteile mit einer Eindringtiefe von $0,25-0,50 \mathrm{~mm}$ geschlitzt werden und damit die Ballonierung effektiver gemacht werden kann. Der eigentliche Dilatationsballon wird über den Port am Katheterende eingeführt. Als Ballongrößen können 2-7 mm Durchmesser $\times 20-120 \mathrm{~mm}$ Länge bei $6 \mathrm{~F}$ und $4-8 \mathrm{~mm} \times 20-40 \mathrm{~mm}$ bei $7 \mathrm{~F}$ genutzt werden. Die Einführkatheter des $\mathrm{XO}$ score ${ }^{\circledR}$ werden mit Längen von $65-140 \mathrm{~cm}$ angeboten.

Die optimierte Kraftübertragung auf die Gefäßwand und die Segmentierung von Plaques durch die oben genannten Hilfsmechanismen bedingt bei suboptimaler Anwendung aber vermehrt Dissektionen, die das primäre Ergebnis in Frage stellen können. Dies kann im Besonderen durch Bewegungen des teilinflatierten Ballons im Blutfluss oder die Dislokation des Ballons im entfalteten Zustand bedingt sein. Um derartige Wandtraumata zu verhindern, wurde ein Scoring-Ballon mit Antischlupfsystem (NSE, „non slip element ${ }^{\prime \prime}$ ) entwickelt (NSE Alpha ${ }^{\circledR}$, B. Braun, Melsungen, Deutschland). Die Studie wurde im vergangenen Jahr publiziert und zeigte in langen femoropolitealen Läsionen deutlich weniger schwere Dissektionen $(8,8 \%$ vs. $29,7 \%, p=0,027)$ eine Verringerung der Dissektionsstrecke $(11,5 \pm 12,8$ vs. $35,7 \pm 24,1 \mathrm{~mm}, p=0,027)$. Dies hatte wiederum einen Effekt auf die Bailout-Stent-Rate in der NSE-Gruppe, die mit $17,6 \%$ vs. $40,5 \%(p=0,035)$ 
Tab. 1 Die Tabelle zeigt vergleichend die Eigenschaften verschiedener Atherektomiesysteme ${ }^{\text {a }}$, die auf dem deutschen Markt angeboten werden. Dabei verweisen die mit + versehenen Felder darauf, welche Eigenschaften oder technischen Besonderheiten vom jeweiligen System vorgehalten werden

\begin{tabular}{|c|c|c|c|c|c|c|c|c|}
\hline & Bycross $^{\circledR}$ & Hawk $^{\circledR}$ family & Laser $^{\circledR}$ & Pantheris $^{\circledR}$ & Rotablator $^{\circledR}$ & Phoenix $^{\circledR}$ & Rotarex $^{\circledR}$ & Jetstream $^{\circledR}$ \\
\hline PACCS 2-4 & + & + & + & + & + & + & - & + \\
\hline CTO & + & - & + & - & + & + & + & + \\
\hline Thrombus & + & - & - & - & - & + & + & + \\
\hline Front + side cutting & + & - & - & - & + & - & - & + \\
\hline Wire rear loading & + & - & + & - & - & - & - & - \\
\hline Dualer Transport & + & - & - & - & - & - & - & + \\
\hline $6 \mathrm{~F} \mathrm{4,7} \mathrm{mm}$ & + & - & - & - & - & - & - & - \\
\hline Drahtlos & + & - & $-{ }^{b}$ & - & - & - & - & - \\
\hline Angio sideport & + & - & - & - & - & - & - & - \\
\hline Variable Kopfgröße & + & - & - & - & - & - & - & - \\
\hline \multicolumn{9}{|c|}{$\begin{array}{l}{ }^{\circledR} \text { Die Hersteller der einzelnen Systeme sind im Folgenden aufgeführt: ByCross }{ }^{\circledR} \text {, Taryag Medical. Inc., Haifa, Israel; Hawk Family }{ }^{\circledR} \text {, Medtronic Inc., Hassett, CA, } \\
\text { USA; B-Laser }{ }^{\circledR} \text {, Eximo Medical, Rehovot, Israel; Pantheris }{ }^{\circledR} \text {, Avinger Inc., Places Redwood City, CA, USA; Rotablator }{ }^{\circledR} \text {, Boston Scientific Inc., Boston, PA, USA; } \\
\text { Rotarex }{ }^{\circledR}, \text { BD and Company, Franklin Lakes, NJ, USA; Jet Stream }{ }^{\circledR} \text {, Boston Scientific Inc., Boston, PA, USA } \\
{ }^{b} \text { Der Laser muss über einen Draht geführt werden, der für den Moment des Energieausstoßes aber in das System zurückgezogen wird }\end{array}$} \\
\hline
\end{tabular}

Tab. 2 Die Tabelle stellt die Läsionsspezifika und die FU-Ergebnisse nach 180 Tagen aus der Zulassungsstudie des ByCross ${ }^{\circledR}$-Systems dar. Daraus ist abzuleiten, dass Atherektomiesysteme mittlerweile auch bei extrem komplexen femoropolitealen Läsionen erfolgreich eingesetzt werden können und damit absehbar eine weitere Herausforderung der Bypasschirurgie erwächst

\begin{tabular}{|c|c|c|c|}
\hline$N$ & 42 & & \\
\hline Alter & 71,9 & $\begin{array}{l}\text { Technologische Erfolgsrate }<30 \% \text { Residu- } \\
\text { alstenose (\%) }\end{array}$ & 95,13 \\
\hline Männlich (\%) & 61,9 & Stentrate (\%) & 28,57 \\
\hline PACSS & 3,1 & Residualstenose im DUS bei Entlassung (\%) & 5,7 \\
\hline Läsionslänge (mm) & 124,7 & 30 Tage MAE & 0 \\
\hline $\begin{array}{l}\text { Referenzgefäß- } \\
\text { diameter }(\mathrm{mm})\end{array}$ & 5,2 & $6 \mathrm{Mo} \mathrm{ABI}$ & 0,85 \\
\hline Stenosegrad prä (\%) & 96,4 & 6 Mo Lumen Loss (\%) & 21,7 \\
\hline $\begin{array}{l}\text { Ruhterford } \\
2 / 3 / 4 / 5(\%)\end{array}$ & $\begin{array}{l}11,9 / 30,9 / 35,7 / \\
21,4\end{array}$ & 6 Mo Primary Patency (\%) & 95,13 \\
\hline
\end{tabular}

deutlich niedriger lag. Die Inflationsdauer und der Inflationsdruck waren nicht signifikant unterschiedlich zwischen den Studiengruppen [35].

\section{DCB ("drug-coated balloon")}

Die kontroverse Diskussion um die Paclitaxel-beschichteten Ballons geht weiter und die Ergebnisse von Studien und Metaanalysen sind widersprüchlich in Bezug auf die erhöhte Spätmortalität, die 2018 von Katsanos et al. publiziert wurden [36]. Die Alternative stellen die Sirolimus-beschichteten Ballons dar, die in seit Anfang 2020 in allen gängigen Größen für die Gefäßperipherie wie auch für AV-Fisteln zur Dialyse zugelassen und greifbar sind. Die wenig gewebegängige Grundsubstanz, das Sirolimus, wird in Verbindung mit Phospholipiden auf den Ballon aufgebracht (Magic
Touch $^{\circledR}$ Sirolimus Ballon, Concept Medical Inc., Tampa, FL, USA) und damit transferierbar.Studienergebnisse dürften in naher Zukunft publiziert werden [37].

\section{Stents}

Insgesamt scheint, hauptsächlich durch Kardiologen und Angiologen vertreten, ein Grundtenor weg vom Implantat vorzuliegen [38].

Allerdings sind Lösungen für Residualstenosen in allen behandelten Gefäßsegmenten notwendig, so dass der Stent, in welcher Form auch immer, weiterhin eine Existenzberechtigung behalten dürfte. Allerdings gibt es auch hier verschiedene Anstrengungen, die Menge an Stentmaterial zu reduzieren und nur flussrelevante Dissektionen per Stent zu behandeln. Das TACK-System (Intact Vascular Inc., Wayne,
PA, USA) mit kurzen, in Reihe in einem kathetergeladenen Nitinolstent soll helfen, dieses Ziel zu erreichen und konnte in den Studien in Kombination mit einem $D C B$ auch gute Ergebnisse erzielen [39, 40].

Allerdings hat die FDA die bisherige Stentindikation, nämlich die mehr als 30\%ige Residualstenose nach PTA entsprechend den IFU (,instructions for use") als Kontraindikation für das System definiert [41], ohne dies transparent darzulegen.

Auf zwei aus unterschiedlichen Gründen interessante Neuerungen möchten die Autoren noch eingehen. Der Jaguar ${ }^{\circledR}$ Stent (Balton, Warschau, Polen), weil er als im Aufbau verwandter direkter Kompetitor zum Supera ${ }^{\circledR}$-Stent (Abbott Global Inc., USA) angesehen werden kann. Zum anderen ein neuer medikamentbeschichteter Nitinolstent, der Amphilimus ${ }^{\circledR}$, eine patentierte Verbindung von Sirolimus mit einer Fettsäure aus kleinen Reservoirs an der abluminalen Seite in das Gewebe abgibt.

Der Jaguar ${ }^{\circledR}$ wird aus einem einzelnen Nitinoldraht gewoben und weist keine Schweißpunkte auf. Er wird in Längen von $20-150 \mathrm{~mm}$ und Durchmessern von 4-14 $\mathrm{mm}$ für den Einsatz über 18er- oder 35er-Drähte bei 6- bis 8-F-Passageprofil angeboten. Die bisher publizierten Daten [42] zeigten eine 5-Jahre-Offenheitsrate in der Poplitea von $31 \%$, dies allerdings bei Patienten mit einem in der Mehrzahl Rutherford-4- bis -5-Stadium und einem pri- 


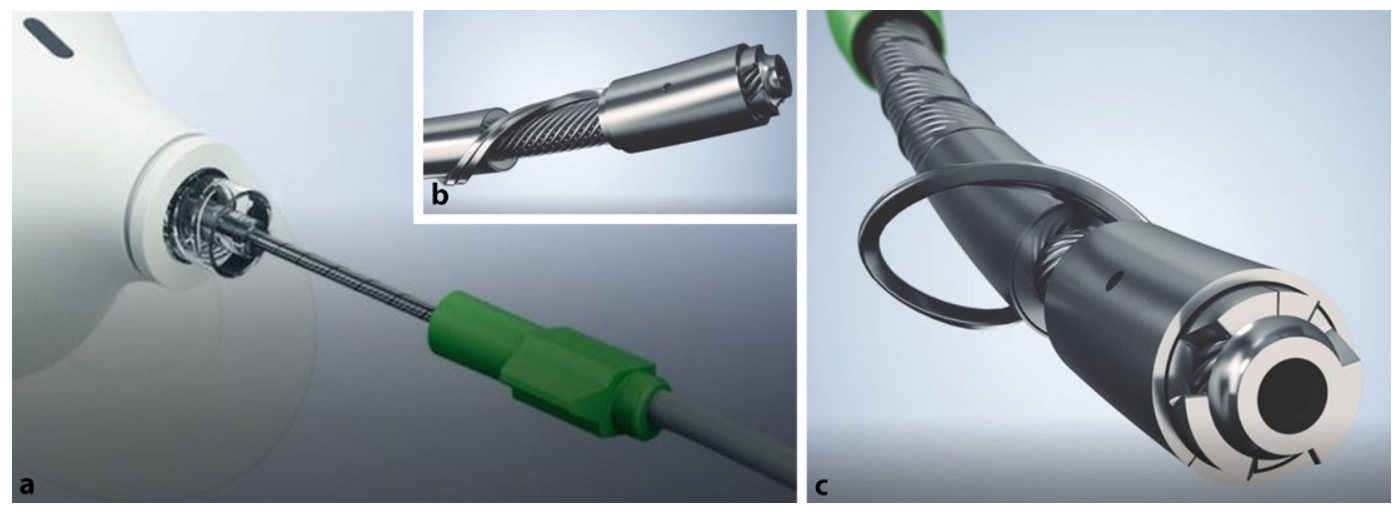

Abb. $6 \Delta$ Besonderheiten des ByCross ${ }^{\circledR}$-Systems: Das System wird in einer am Handgriff befestigten 6-F-Schleuse (a) in das Zielgefäß vorgeschoben, über die die im Handgriff untergebrachte Ansaugpumpe zeitgleich mit dem unterhalb der Spitze porösen Schaft (b), der dem Prinzip der Archimedischen Schraube folgt, Material aspiriert und transportiert. Der Bohrkopf hat einen variablen Durchmesser. Dieser misst zwischen 1,9 mm (b) und 4,7 mm bei ausgefahrenem Nitinolschneidedraht (c). (Mit freundl. Genehmigung Taryag Medical Inc., Israel)

mär bereits reduzierten Run-off als Negativmerkmale. So lag die 5-Jahre-Offenheitsrate bei Patienten mit zwei bzw. drei Run-off-Gefäßen bei $61 \%$ bzw. $68 \%$. Auch bei diesem gewobenen Nitinolstent zeigte sich die Bruchresistenz im maximal mobilen Gefäßsegment. Stentfrakturen traten im benannten Nachbeobachtungszeitraum nicht auf.

Der NiTiDES ${ }^{\circledR}-$ Stent (Alvimedica, Istanbul, Türkei) ist ein Nitinolstent mit Carbonbeschichtung und Reservoirs auf der $\mathrm{Au}$ Benseite, über die die Wirksubstanz über einen längeren Zeitraum abgegeben wird. Die Kombination von Sirolimus und Fettsäure führt dabei zu einer homogenen Verteilung im Gewebe. Sirolimus hat den Vorteil der stark antiproliferativen und antiinflammatorischen Wirkung [43] bei einer gegenüber dem Paclitaxel deutlich geringeren Toxizität [44].

In der multizentrischen ILLUMINA-Studie mit einer Nachbeobachtungszeit von 24 Monaten zeigte sich eine Offenheitsrate von $89,3 \%$ nach 12 und von $83,4 \%$ nach 24 Monaten. Die Revaskularisierungsrate lag lediglich bei $6,9 \%$ (freedom from TLR ["target lesion revascularization"] $93,1 \%$ ) bei einer Läsionslänge von $72,54 \pm 37,99 \mathrm{~mm}[45]$.

\section{Atherektomiesysteme}

Stark kalzifizierte Läsionen stellen weiterhin die größte Herausforderung für endovaskuläre Vorgehen dar. Im Besonderen dann, wenn diese Läsion langstreckig und exzentrisch oder als Ausgusspräparat die
Wand infiltrierend das gesamte Gefäßlumen ausfüllen.

Die endovaskulären Optionen sind zwar auch hier mannigfaltig, aber mehr oder weniger aufwendig, mit mehreren Gefäßzugängen behaftet, teilweise sehr kostenintensiv und wesentlich vom Erfahrungsgrad des Anwenders (Operateur und Team) abhängig. Eine Möglichkeit, schwer kalzifizierte Läsionen zu passieren oder passierbar zu machen, ist die Pave-and-crackTechnik, die bereits 2007 von Hinchcliff et al. aus der Malmö'er Arbeitsgruppe um Martin Malina als robuste Technik vorgestellt wurde, um Beckenarterienstenosen für Aortenendografts passierbar zu machen [46].

\section{I) Stark kalzifizierte Läsionen stellen die größte Herausforderung für endovaskuläre Vorgehen dar}

Im femoropoplitealen Segment versteht man darunter die subintimale Passage von Läsionen, bei der gezielt Wandstrukturen zerstört und anschließend zur Verhinderung von Blutungen gecoverte Stents eingebracht werden, die letztendlich im subintimalen Raum auf ihren Nominaldurchmesser dilatiert werden [47]. Dabei spielt es keine Rolle, ob ein Draht primär von proximal oder distal die Stenose passiert. Allerdings ist diese Methode nur einsetzbar, wenn noch keine Implantate vor Ort sind.

Die endovaskuläre, kathetergestützte Entfernung von Plaquesmaterial mit scharfen Instrumenten ist als therapeutischer
Ansatz wieder in den Mittelpunkt des Interesses gerückt. Das kann daran liegen, dass neue Systeme auf den Markt gekommen sind oder dass die Hersteller dem Wunsch der Kliniker Folge geleistet haben, robuste Systeme für die Behandlung kalzifizierter Gefäßstrecken anzubieten. Mittlerweile ist die Bandbreite der Systeme groß geworden und sowohl für den Unterschenkel wie für die femoropopliteale und koronare Gefäßstrecke sind diese Materialien anwendbar. Allerdings unterscheiden sie sich wesentlich in der praktischen Anwendung und der Logistik dahinter (• Tab. 1).

Logistik bedeutet in diesem Fall die Unterscheidung zwischen "disposable", also einem meist batteriebetriebenem Einmalsystem ohne Investition, oder einem Kathetersystem, das an eine Motoreinheit angeschlossen wird, die als Investitionsgut angeschafft werden muss. Zudem werden einige Systeme in mehreren Größen (Bohrkopfdurchmessern) und Passageprofilen angeboten. Andere haben variable Kopfgrößen, die den Einsatz in unterschiedlichen Gefäßdurchmessern und die Schaffung eines größeren Lumens mit nur einem System erlauben. Die Ergebnisse der Studien sind vielversprechend, dies auch für lange Läsionen, die bisher eher der Bypasschirurgie vorbehalten waren.

Für das Phoenix ${ }^{\circledR}$-System (Philips b.v., Eindhoven, Niederlande) zeigt sich eine technische Erfolgsragte von $>96 \%$ für Läsionen zwischen 5 und $23 \mathrm{~cm}$ und 1-, 6- und 12-Monats-Offenheitsraten von $96,1,86,5$ und $76,9 \%$ und einer assistierten primären Offenheitsrate nach 1, 6 und 
12 Monaten von $100,90,3$ und $86,5 \%$ [7]. Andere Systeme, z.B. das ByCross ${ }^{\circledR}$ System (Taryag Medical Inc., Haifa, Israel), das sich in der CE-Studie (ClinicalTrials.gov NCT03724279) gerade bei der Therapie stark verkalkter Läsionen als besonders geeignet erwiesen hat, ist mit einer Vielzahl an Drähten unterschiedlicher Dicke und Beschichtung kompatibel. Das System kann zudem ohne primäre Drahtpassage im Sinne eines Rekanalisierungssystems eingesetzt werden (• Abb. 6). In der CEStudie zeigte sich für Läsionen zwischen 30-450 (im Mittel 124,7) mm eine technische Erfolgsrate von 95,12\% bezogen auf eine angiographische Residualstenose nach Atherektomie von $<50 \%$ und $<30 \%$ nach Komplettierung der Prozedur bei Patienten im Rutherford-Stadium 2-5 (siehe - Tab. 2).

Aktuell läuft eine Registerstudie mit einem Laseratherektomiesystem, dem Auryon ${ }^{\circledR}$ [48], Angiodynamics, Saratoga Springs, NY, USA. Hierbei handelt es sich um einen 355-nm-Laser. Diese als Feststofflaser bezeichneten Systeme konnten eine hohe Effektivität hinsichtlich der Desintegration verschiedenster Materialien nachweisen [49]. Im Rahmen der Studie sollen De-novo-Stenosen, Restenosen und In Stentstenosen der infrainguinalen Gefäße behandelt werden.

Für die Behandlung komplexer Gefäßläsionen im AFS-Segment stellt das PQ Bypass ${ }^{\circledR}$-Verfahren von PQ Bypass Inc. Dublin, Irland, eine Alternative dar, die in der DETOUR-Studie interessante Ergebnisse in der Hand des erfahrenen Interventionalisten nachweisen konnte [50]. Die durchschnittliche Läsionslänge lag bei $370 \mathrm{~mm}$ mit $96 \%$ chronischer Verschlüsse und mäßiger bis schwerer Kalzifizierung bei $81 \%$ der Läsionen. Die primäre 12-Monats-Offenheitsrate lag bei $71 \%$, die primär assistierte Offenheitsrate bei $80 \%$. Bei diesem Verfahren werden gecoverte Stents von der AFS zunächst in die tiefe Vene und distal des Verschlusses wieder in die Zielarterie geführt. Durch die Implantation von gecoverten Stents sind die Kosten für das Verfahren hoch anzusetzen und die Kosteneffektivität muss noch evaluiert werden.

Das Limflow ${ }^{\circledR}$-Kathetersystem (LimFlow SA, Paris, Frankreich) dient der perkutanen retrograden Arterialisierung bei arteriell nicht rekonstruierbaren Gefäßstrecken [51]. Der Zugang erfolgt perkutan antegrad zur Arterie und von distal retrograd über die Zielvene. Wie bei der offen chirurgischen retrograden Arterialisierung müssen die Venenklappen der abführenden Venen zerstört werden. Dazu wird ein antegrad wirkendes 4-F-Valvulotom angewendet. Teilweise werden langstreckig gecoverte Stents in die Venen eingesetzt, um das tiefe Venensystem zu arterialisieren. Das Prinzip wurde in der Koronarchirurgie bereits in den 1970erJahren erfolgreich bei selektierten Patienten eingesetzt [52] und lässt auch bei jungen Patienten mit Thrombendangiitis obliterans und den dann typischen distalen und akralen Verschlussmorphologien Optionen zum Extremitätenerhalt offen.

\section{Diskussion}

Seit der Erstbeschreibung einer Gefäßdilatation durch Dotter 1964 [53] hat die endovaskuläre Therapie in wesentlichen gefäßtherapeutischen Bereichen eine dominante Rolle eingenommen. Sowohl die Entwicklung der Materialien wie auch die Optimierungen der begleitenden medikamentösen Therapie haben diese Entwicklung unterstützt. Diese Dominanz spiegelt sich noch auffälliger in der medialen Präsenz wieder. Die gefäßmedizinischen Fachgesellschaften haben sich dieser Entwicklung durch die Veränderung der Ausbildungsinhalte angepasst [54].

Die Innovation geriet zwar durch die SARS-CoV-2-bedingten ("severe acute respiratory syndrome coronavirus 2") Umstände ins Stocken, verschiedene Studien zu neuen Materialien sind aber publiziert oder bereits auf virtuellen Kongressen vorgestellt worden.

Die hier vorgestellten Systeme und Studienergebnisse untermauen den seit Jahren bestehenden Trend, durch die Verbesserung des Materials und des breitflächigen Zugriffs auf diese Systeme die endovaskuläre Versorgung anatomisch und histologisch komplexer Verschlussmorphologien zu ermöglichen. Damit fällt die rekonstruktive Gefäßchirurgie im aortoiliakalen und femoropolitealen Bereich wahrscheinlich weiter ins Hintertreffen. Dies entspricht nicht mehr der 2015 veröffentlichten Leitlinie zur Behandlung der pAVK [55], aber die Entwicklung ist nicht aufzuhalten. Ob damit auch für diesen Bereich der Gefäßtherapie eine Zentralisierung einhergehen wird, bleibt abzuwarten.

Die aktuellen Entwicklungen der Versorgungsqualität im chirurgischen Bereich sprechen leider auch für das endovaskuläre Verfahren. Seit 2005 die BASIL-Studie und die Langzeitdaten veröffentlicht wurden [1, 2], hat sich die Ergebnisqualität der Bypasschirurgie zumindest in Großbritannien sogar verschlechtert. Bei statistisch signifikant niedrigerer 30-Tage-Morbidität und -Mortalität ( $22 \%$ vs. $45 \%$ in BASIL, $p=0,005)$ und nicht-signifikant unterschiedlichen Bollinger- und IP-GLASSScores lag das amputationsfreie Überleben bei nur $28 \%$ gegenüber $62 \%$ in BASIL $(p=0,007)$. Die Beinerhaltungsrate sank von $85 \%$ in BASIL auf $69 \%$ $(p=0,02)$ und die Überlebensrate von $69 \%$ auf $35 \%(p=0,05)$. Auch die Rate der MALE („major adverse limb events") stieg von $47 \%$ in BASIL auf $67 \%$ in der aktuellen Analyse ( $p=0,01 ;[3])$. Ob dies Folge einer negativen Selektion oder eines Kompetenzverlusts ist, ist aus den Daten nicht ableitbar. Die deutlich geringere Morbiditäts- und Mortalitätsrate in der beobachteten Patientengruppe spräche eher für verbesserte Anästhesieverfahren und weniger komplikationsbehaftete Eingriffe, also gegen den Kompetenzverlust.

Die Aufarbeitung der IDOMENEODaten dürfte einen tieferen Einblick in das gefäßtherapeutische Versorgungsgeschehen in Deutschland geben (https:// innovationsfonds.g-ba.de/projekte/ versorgungsforschung/idomeneo-studieist-die-versorgungsrealitaet-in-dergefaessmedizin-leitlinien-undversorgungsgerecht; [8]).

\section{I) Die Schattenseite der endovaskulären, Röntgen-basierten Maßnahmen ist die höhere Strahlenbelastung}

Die weitere Entwicklung neuer Systeme und deren Marktzulassung haben durch die Änderungen der europäischen Vorgaben in Zukunft sicherlich eine höhere Hürde zu nehmen. Aber die schon zertifizierten Materialien erlauben bereits jetzt eine nahezu komplette Abdeckung der rekonstruktiven Herausforderungen. Dies 
reicht bis zur retrograden Arterialisierung als letzte Option des Extremitätenerhalts $\left(\right.$ LimFlow $\left.^{\circledR}\right)$ bei fehlenden arteriellen Rekonstruktionsmöglichkeiten.

Die Schattenseite der Ausweitung endovaskulärer, Röntgen-basierter Maßnahmen ist die höhere Strahlenbelastung für Operateur, Team und Patient.

Mit der wachsenden Komplexität der Prozeduren, unabhängig davon, ob sie mit Pave-and-crack-Technik, speziellen Ballons oder Atherektomiesystemen durchgeführt werden, dürfte die Strahlenbelastung für die einzelne Operation und alle Anwesenden zunehmen. Gerade dann, wenn Operateur und Strahlenquelle in enger Nachbarschaft positioniert sind und passiver Strahlenschutz am Tisch den Zugang zum Gefäß erschwert.

Damit fällt der Reduktion der Strahlungsgenerierung in der einzelnen Prozedur die wichtigste Rolle zu. Ultraschallgesteuerte Maßnahmen können die Strahlendosis signifikant reduzieren. Dies beginnt mit der ultraschallgesteuerten und nicht Röntgen-gesteuerten Punktion, unabhängig vom Gefäßzugang.

Mittels IVUS kann eine dreidimensionale, hochauflösende Bildgebung von Gefäßen erfolgen, die eine Angiographie redundant macht und damit die Strahlendosis reduziert [11]. Gleichzeitig kann IVUS die Bildgebung sicherer und genauer machen, und dies nicht nur im aortalen Bereich $[12,14]$.

Bei der endovaskulären Versorgung venöser Läsionen steht die Überlegenheit des IVUS nicht mehr zur Debatte [16]. Die Detektionssicherheit für Stenosen und die Aussagekraft hinsichtlich der zu erwartenden Langzeitergebnisse der Prozeduren sind für das IVUS ungleich höher [17, 18].

Im arteriellen Bereich scheint die Perzeption dieser Technik noch wenig ausgeprägt, obwohl auch für dieses wie für das koronare Segment Daten vorliegen, die den Einsatz aufgrund der besseren Gesamtergebnisse und des Patientenoutcomes mit deutlich geringerem Risiko unterstützen [19-21].

Gerade technische Hilfsmittel (die das Arbeiten sicherer und risikoärmer machen) wie IVUS oder steuerbare Materialien gewinnen an Bedeutung, wenn mehr Interventionen durchgeführt werden und sie damit das Arbeitsleben positiv beeinflussen. Strahlenschutz muss für alle Beteiligten dauerhaft von hohem Interesse sein und ist ja auch durch die Strahlenschutzgesetzgebung zur Pflicht gemacht worden. Mit dem Einzug der virtuellen Realität in den operativen Bereich dürfte die Kombination von FORS und IVUS eine interessante Alternative für Patienten und Mitarbeiter darstellen, die neben dem Faktor Strahlenschutz auch den Faktor Mitarbeiterbindung unterstützen kann [9]. FORS kann zwar die Durchleuchtungszeit beeinflussen, eine Darstellung des Gefäßlumens und der Rekonstruktionsergebnisse ist aber weiterhin nur mit der Gabe von Kontrastmittel oder der Anwendung von IVUS als komplementäre Technik möglich.

Die Anwendung spezieller, kleinlumiger Schleusen zum retrograden Zugang an den Unterschenkelgefäßen, die mittlerweile breitflächig als zusätzlicher oder alleiniger Zugang für komplexe Läsionen genutzt werden, vereinfacht die Prozedur und erlaubt die angiographische Kontrolle über die distale Schleuse. Damit könnte der Rendezvouszugang femoral entfallen und der Strahlenschutz verbessert werden. Dies wäre eine weniger traumatische sowie Zeit und Kosten einsparende Vorgehensweise, da ein zweiter arterieller Zugang samt dem erforderlichen Materialverbrauch entfiele.

Die Weiterentwicklung von Carrier-Systemen, wie Drähten und Schleusen, und den eigentlich therapeutischen Modulen, den Ballons oder Atherektomiesystemen, sollte es auch ermöglichen, mit weniger Kontrastmittel- und Strahlendosis effektiv zu arbeiten.

Die Vielfalt der Systeme mit unterschiedlichen Anbietern verlangt vom Anwender ein intensives Auseinandersetzen mit diesen Materialien, um diese professionell einsetzen zu können. Unabhängig von der Wahl des Materials und der Methode ist bei all diesen komplexen Interventionen bereits bei der Planung darauf zu achten, dass die Einzelkomponenten miteinander kompatibel sind, um technisch erfolgreich zu sein. Die Materialtestungen der verschiedenen Hersteller zielen nicht darauf ab, die Systemkompatibilität mit Materialien anderer Anbieter zu beweisen. Die Dickenmaße von Drähten und die Innenmaße von Kathetern sind nur Richtwerte, keine DIN-Werte. Damit obliegt es dem Anwender, sein Portfolio so zusammen zu stellen, dass Inkompatibilitäten auftreten.

Die angeführten Systeme zielen in ihrer Gesamtheit darauf ab, komplexe Läsionen, die bisher vielleicht noch die Domäne der Bypasschirurgie waren, endovaskulär effektiv therapierbar zu machen. Dabei sind die schwer kalzifizierten Läsionen die aktuelle, vielleicht auch letzte Herausforderung, gerade wenn es sich um TASC-IIC- und -D-Läsionen handelt. Die klinische Realität hält sich nicht mehr an die Vorgaben der AWMF-Leitlinie von 2015 (Arbeitsgemeinschaft Medizinisch Wissenschaftlicher Fachgesellschaften).

Das wachsende Interesse an der Atherektomie auf Seiten der Anwender wie auch der Industrie und die Vielfalt der auf dem Markt befindlichen Systeme reflektieren die Anstrengungen, auch bisher als dem Bypassverfahren vorbehaltene Gefäßläsionen in allen Segmenten endovaskulär anzugehen. Die zuletzt veröffentlichten Ergebnisse des Phoenix ${ }^{\circledR}$-Systems (Philips b.v., Niederlande) oder die 6-Monats-Offenheitsraten der CE-Studie zum ByCross ${ }^{\circledR}$ System (Taryag Medical Inc., Israel) stehen den offen chirurgischen Ergebnissen in Nichts nach. Die Invasivität der Eingriffe dürfte bei Anwendung von Lokalanästhesie jedoch deutlich geringer gewesen sein, als das chirurgische Verfahren und dies mit deutlich kürzeren Liegezeiten für die Patienten. Daher ist die intensive Beschäftigung mit dem endovaskulären Sektor und den sich entwickelnden Möglichkeiten unabdingbar, ohne den offen chirurgischen Möglichkeiten den Rücken zu kehren.

Durch die Fokussierung der Systementwicklung in Richtung komplexer Läsionen und mobiler Gefäßsegmente ist der Begriff "Gefäßpräparation" ins Zentrum des Interesses gerückt.Zum einen, um ein Implantat entsprechend den spezifischen Anforderungen implantieren zu können (Überdilatation der Gefäßwand vor Implantation eines Superastents ${ }^{\circledR}$ [Abbott Global Inc., USA]). Zum anderen, um durch den Einsatz speziell designter Systeme die Zahl der notwendigen Implantate zu reduzieren oder diese gänzlich redundant zu machen. Hier sei das TACK-System (Intact vascular Inc., USA) zu nennen oder die Atherektomie, die über die Resektion oder Verdampfung 
(Laser) des Verschlussmaterials ein ausreichendes Gefäßlumen, ggf. in Verbindung mit Ballondilatationen und ohne Stentimplantat schaffen kann. In der CE-Studie für das ByCross ${ }^{\circledR}$-System lag bei Verschlusslängen von durchschnittlich 124 (30-450) $\mathrm{mm}$ die Rate der Nachdilatation bei $94 \%$, die Stentrate bei $28 \%$. Außerdem wurde in keinem der Fälle ein Embolieprotektionssystem benötigt. Je nach Wahl des Rekonstruktionsverfahrens können immense Kostenunterschiede entstehen, die unter dem allgegenwärtigen Kostendruck das Vorgehen mit beeinflussen dürften.

Hinsichtlich der Drug-eluting-Technologien müssen die sehr guten Ergebnisse der neueren Sirolimus-beschichteten Ballon- und Stentsysteme durch größere Studien validiert werden $[37,45]$. Sirolimus unterliegt erfreulicherweise auch nach Jahren des Einsatzes nicht dem Verdacht der systemischen Toxizität und der Substanz wird nicht unterstellt, die Spätmortalität negativ zu beeinflussen [44].

\section{Fazit für die Praxis}

Für die Chirurgie und den einzelnen Gefäßchirurgen bleibt angesichts dieser Entwicklungen die Aufgabe, sich bereits während der Ausbildung mit den erprobten Materialien und mit den neuen Techniken intensiv zu beschäftigen aber gleichzeitig die Ergebnisqualität der Bypasschirurgie und die chirurgische Ausbildung nicht zu vernachlässigen. Diesen Spagat schmerzfrei vorzuführen wird schwierig sein.

Aber möglicherweise wird sich nun auf diesem Gebiet der Gefäßtherapie die nächste Zentralisierung von komplexen Leistungen anbahnen. Denn komplexe endovaskuläre Verfahren erfordern im Falle einer Komplikation auch das Vorhalten des differenzierten chirurgischen „bail-out“ mit Lösungen für komplexe Probleme unter ungünstigen Ausgangsbedingungen.

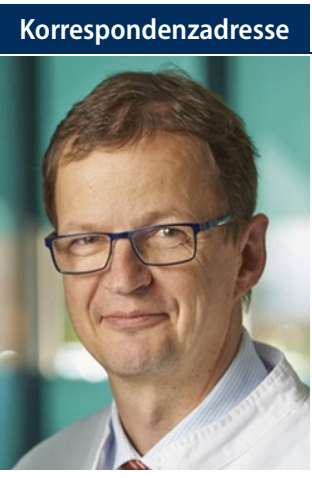

\section{Dr. Jörg Teßarek}

Klinik für Gefäßchirurgie, Bonifatius Hospital Lingen

Wilhelmstraße 13, 49808 Lingen, Deutschland joerg.tessarek@hospital-lingen.de

\section{Einhaltung ethischer Richtlinien}

Interessenkonflikt. J. Teßarek weist auf folgende Beziehungen hin: Dr. Teßarek war PI der Zulassungsstudie des ByCross Systems und hat in diesem Jahr Vortragshonorare der Firmen plusmedica GmbH und Co. KG sowie von Philips Niederlande erhalten. A. Oberhuber weist auf folgende Beziehungen hin: Forschungsförderung von Altura Medical, Endologix, Jotex, Bayer, Endospan, Medyra, GELITA, Cordis. Reise-/Übernachtungskosten von Cook, Jotec, Lombard, Medtronic, Gore, Endologix, Bayer, Pluristem, PQ Bypass; Vortragshonorare von Jotec, Lombard, Endologix, Medtronic, Gore. Tätigkeit für Lombard (Workshopleiter), Endologix (Proctor und Workshopleiter), Jotec (Proctor).

Für diesen Beitrag wurden von den Autoren keine Studien an Menschen oder Tieren durchgeführt. Für die aufgeführten Studien gelten die jeweils dort angegebenen ethischen Richtlinien.

\section{Literatur}

1. Adam DJ, Beard JD, Cleveland T, Bell J, Bradbury AW, Forbes JF et al (2005) Bypass versus angioplasty in severe ischaemia of the leg (BASIL): multicentre, randomised controlled trial. Lancet 366(9501):1925-1934

2. Bradbury AW, Adam DJ, Bell J, Forbes JF, Fowkes FGR, Gillespie I et al (2010) Bypass versus Angioplasty in Severe Ischaemia of the Leg (BASIL) trial: An intention-to-treat analysis of amputation-free and overall survival in patients randomized to a bypass surgery-first or a balloon angioplasty-first revascularization strategy. J Vasc Surg 51:5S-17S

3. Meecham L, Popplewell M, Bate G, Patel S, Bradbury AW (2019) Contemporary (2009-2014) clinical outcomes after femoropopliteal bypass surgery for chronic limb threatening ischemia are inferior to those reported in the UK Bypass versus Angioplasty for Severe Ischaemia of the Leg (BASIL) trial (1999-2004). JVasc Surg 69(6):1840-1847

4. Lichtenberg M, Korosoglou G (2019) Atherectomy plus antirestenotic therapy for SFA lesions: evolving evidence for better patency rates in complex lesions. J Cardiovasc Surg 60(2):205-211
5. Sauguet A, Philippart R, Honton B (2019) Directional atherectomy with antirestenotic therapy for the treatment of no-stenting zones. JCardiovasc Surg 60(2):198-204

6. Goueffic Y, Schiava DN, Thaveau F, RossetE, Favre JP, du Mont SL et al (2017) Stenting or surgery for de novo common femoral artery stenosis. JACC Cardiovasc Interv 10(13):1344-1354

7. Gandini R, Pratesi G, Merolla S, Scaggiante J, Chegai $F$ (2020) A single-center experience with phoenix atherectomy system in patients with moderate to heavily calcified femoropopliteal lesions. Cardiovasc Revasc Med 21(5):676-681

8. Teßarek J (2020) 6 Monatsergebnisse der ByCross Atherektomie CE Zulassungstudie. LINC, Leipzig

9. van Herwaarden JA, Jansen MM, Vonken EPA, Bloemert-Tuin T, Bullens RWM, de Borst GJ et al (2021) First in human clinical feasibility study of endovascularnavigation with fiber optic realshape (FORS) technology. Eur J Vasc Endovasc Surg 61(2):317-325

10. Floros N, Schelzig H, Kölbel T, Schmidt A, Branzan D, Kalder Jetal (2019) First-in-man clinical application of the trackcath system in endovascular repair of aortic aneurysms; a prospective multicentre clinical trial. Eur J Vasc Endovasc Surg 58(6):e723-e724

11. TeßarekJ (2021) IVUS in EVAR. EVC, Maastricht

12. Lortz J, Papathanasiou M, Rammos C, Steinmetz M, Lind A, Tsagakis K et al (2019) High intimal flap mobility assessed by intravascular ultrasound is associated with better short-term results after TEVAR in chronic aortic dissection. Sci Rep 9(1):7267

13. Lortz J, Tsagakis K, Rammos C, Horacek M, Schlosser T, Jakob H et al (2018) Intravascular ultrasound assisted sizing in thoracic endovascular aortic repair improves aortic remodeling in Type B aortic dissection. PLoSONE 13(4):e196180

14. Pliagas G, Saab F, Stavroulakis K, Bisdas T, Finton S, Heaney $C$ et al (2020) Intravascular ultrasound imaging versus digital subtraction angiography in patients with peripheral vascular disease. JInvasive Cardiol 32(3):99-103

15. Raju S, Buck WJ, Crim W, Jayaraj A (2018) Optimal sizing of iliac vein stents. Phlebology 33(7):451-457

16. Montminy ML, Thomasson JD, Tanaka GJ, Lamanilao LM, Crim W, Raju S (2019) A comparison between intravascularultrasound and venography in identifying key parameters essential for iliac vein stenting. J Vasc Surg Venous Lymphat Disord 7(6):801-807

17. Gagne PJ, Tahara RW, Fastabend CP, Dzieciuchowicz L, Marston W, Vedantham S et al (2017) Venography versus intravascular ultrasound for diagnosing and treating iliofemoral vein obstruction. J Vasc Surg Venous Lymphat Disord 5(5):678-687

18. Gagne PJ, Gasparis A, BlackS, Thorpe P, Passman M, Vedantham S et al (2018) Analysis of threshold stenosis by multiplanar venogram and intravascular ultrasound examination for predicting clinical improvement after iliofemoral vein stenting in the VIDIO trial. J Vasc Surg Venous Lymphat Disord 6(1):48-56e1

19. Buckley CJ, Arko FR, Lee S, Mettauer M, Little D, Atkins $M$ et al (2002) Intravascular ultrasound scanning improves long-term patency of iliac lesions treated with balloon angioplasty and primary stenting. JVasc Surg 35(2):316-323

20. Sheikh AB, Anantha-Narayanan M, Smolderen KG, Jelani QU, Nagpal S, Schneider Met al (2020) Utility of intravascular ultrasound in peripheral vascular 
interventions: systematic review and metaanalysis. Vasc Endovascular Surg 54(5):413-422

21. Darmoch F, Alraies MC, Al-Khadra Y, Moussa Pacha H, Pinto DS, Osborn EA (2020) Intravascular ultrasound imaging-guided versus coronary angiography-guided percutaneous coronary intervention: a systematic review and metaanalysis. J Am Heart Assoc 9(5):e13678

22. Usai MV, Oberhuber A, Asciutto G (2020) Assessment of bridging stent grafts in branched endovascular aortic repair (EVAR) procedures using intravascular ultrasound (IVUS). EJVES Vasc Forum 47:51-54

23. Michael TT, Alomar M, Papayannis A, Mogabgab O, Patel VG, Rangan BV et al (2013) A randomized comparison of the transradial and transfemoral approaches for coronary artery bypass graft angiography and intervention: the RADIAL-CABG Trial (RADIAL Versus Femoral Access for Coronary Artery Bypass Graft Angiography and Intervention). JACC Cardiovasc Interv6(11):1138-1144

24. Aoun J, Hattar L, Dgayli K, Wong G, Bhat T (2019) Update on complications and their management during transradial cardiac catheterization. Expert Rev Cardiovasc Ther 17(10):741-751

25. Harvey JA, Kim S, Ireson ME, Gulati R, Bell MR, Moran SL (2020) Acute upper-limb complications following radial artery catheterization for coronary angiography. JHand Surg Am 45(7):655e1-655e5

26. Ruzsa Z, Bellavics R, Nemes B, Huttl A, Nyerges A, Sotonyi $P$ et al (2018) Combined transradial and transpedal approach for femoral artery interventions. JACC Cardiovasc Interv 11(11):1062-1071

27. El-Sayed H, Bennett ME, Loh TM, Davies MG (2016) Retrograde pedal access and endovascular revascularization: a safe and effective technique for high-risk patients with complex tibial vessel disease. Ann Vasc Surg 31:91-98

28. Kassimis G, Kontogiannis N, Raina T (2019) Steerable microcatheters for complex percutaneous coronary interventions in octogenarians: from Venture to Swift Ninja.J Geriatr Cardiol 16(1):54-59

29. Hinrichs JB, Marquardt S, Wacker FK, Meyer BC (2017) Coil embolization of reversed-curve hepatointestinal collaterals in radioembolization: potential solutions for a challenging task. Radiol Case Rep 12(3):529-533

30. Gandini R, Fabiano S, Spano S, Volpi T, Morosetti D, Chiaravalloti A et al (2013) Randomized control study of the outback LTD reentry catheter versus manual reentry for the treatment of chronic total occlusions in the superficial femoral artery. Catheter Cardiovasc Interv 82(3):485-492

31. Diamantopoulos A, Santonocito S, Thulasidasan N, Gkoutzios P, Ahmed I, Zayed H et al (2018) Initial experience with the outback catheter for targeted reentry during subintimal angioplasty of the infragenicular arteries. J Endovasc Ther 25(2):178-182

32. Patrone L, Stehno O (2019) Retrograde insertion of the outback reentry device from a tibial artery for complex infrainguinal recanalization. CVIR Endovasc 2(1):47

33. Horie K, Tanaka A, Taguri M, Kato S, Inoue N (2018) Impact of prolonged inflation times during plain balloon angioplasty on angiographic dissection in femoropopliteal lesions. J Endovasc Ther 25(6):683-691

34. Holden A, Hill A, Walker A, Buckley B, Merrilees S, Nowakowski P et al (2019) PRELUDE prospective study of the Serranator device in the treatment of atherosclerotic lesions in the superficial femoral and popliteal arteries. J Endovasc Ther 26(1):18-25

35. Karashima E, Yoda S, Yasuda S, Kajiyama S, Ito H, Kaneko T (2020) Usefulness of the "non-slip element" percutaneous transluminal angioplasty

\section{Innovations in the endovascular treatment of peripheral arterial disease}

The cooperation of physicians, engineers and other sciences results in a steady launching of effective tools for vascular treatment, which further support the dominating position of endovascular therapy in the field of PAD. Parallel to the treatment options, non-radiation based image guidance is another area, where innovation helps to reduce radiation burden for patient and staff members without losing procedural quality and despite a growing number of radiation based procedures. Meanwhile, the available portfolio of endovascular tools allows to treat every vessel segment with results comparable to or even better then open surgery. This survey presents new techniques, tools and recently published results from related trials, which will probably have further influence on PAD treatment in the coming years.

\section{Keywords}

Atherectomy · Critical limb ischemia · Stenosis · Catheterization · Angioplasty

balloon in the treatment of femoropopliteal arterial lesions. J Endovasc Ther 27(1):102-108

36. Katsanos K, Spiliopoulos S, Kitrou P, Krokidis M, Karnabatidis D (2018) Risk of death following application of paclitaxel-coated balloons and stents in the femoropopliteal artery of the leg: a systematic review and meta-analysis of randomized controlled trials. J Am Heart Assoc 7(24):e11245

37. Ono M, Kawashima H, Hara H, Katagiri Y, Takahashi $\mathrm{K}$, Kogame $\mathrm{N}$ etal (2020) A prospective multicenter randomized trial to assess the effectiveness of the magic touch sirolimus-coated balloon in small vessels: rationale and design of the TRANSFORM I trial. Cardiovasc Revasc Med 25:29-35

38. Banerjee $\mathrm{S}$, Jeon-Slaughter $\mathrm{H}$, Armstrong EJ, Bajzer C, Abu-Fadel M, Khalili H et al (2019) Clinical outcomes and cost comparisons of stent and nonstent interventions in infrainguinal peripheral artery disease: insights from the excellence in peripheral artery disease (XLPAD) registry. J Invasive Cardiol 31(1):1-9

39. Gray WA, Cardenas JA, Brodmann M, Werner M, Bernardo NI, George JC et al (2019) Treating postangioplasty dissection in the femoropopliteal arteries using the tack endovascular system: 12-month results from the TOBA II study. JACC Cardiovasc Interv 12(23):2375-2384

40. Brodmann M, Wissgott C, Brechtel K, Nikol S, Zeller T, Lichtenberg M et al (2020) Optimized drug-coated balloon angioplasty of the superficial femoral and proximal popliteal arteries using the Tack Endovascular System: TOBA III 12-month results. JVasc Surg 72(5):1636-1647.e1

41. FDA Tack endovascular system ${ }^{\circledR}(6 F)-P 180034$. https://www.fda.gov/medical-devices/recentlyapproved-devices/tack-endovascular-systemr6f-p180034.Zugegriffen: 5. Jan. 2019

42. Takacs T, Mihalovits G, Varadi R, Palasthy Z (2020) Early experience with self-expanding flexible stent for the treatment of atherosclerotic diseases in the popliteal artery. Orv Hetil 161(15):588-593

43. Parry TJ, Brosius R, Thyagarajan R, Carter $D$, Argentieri D, Falotico R et al (2005) Drug-eluting stents: sirolimus and paclitaxel differentially affect cultured cells and injured arteries. Eur J Pharmacol 524(1-3):19-29

44. Wessely R, Schomig A, Kastrati A (2006) Sirolimus and paclitaxel on polymer-based drug-eluting stents: similar but different. J Am Coll Cardiol 47(4):708-714

45. Scheinert D (2020) Innovative siroLimus self Expanding drUg-eluting stent for the treatMent of perlpheral disease: evaluation of safety aNd
efficAcy. The ILLUMINA Study. (ILLUMINA). LINC, Leipzig

46. Hinchliffe RJ, Ivancev K, Sonesson B, Malina M (2007) "Paving and cracking": an endovascular technique to facilitate the introduction of aortic stent-grafts through stenosed iliac arteries. JEndovasc Ther 14(5):630-633

47. Dias-Neto M, Matschuck M, Bausback Y, BanningEichenseher U, Steiner S, Branzan D et al (2018) Endovascular treatment of severely calcified femoropopliteal lesions using the "pave-andcrack" technique: technical description and 12month results. J Endovasc Ther 25(3):334-342

48. (2021) Post-market registry of Auryon ${ }^{\mathrm{TM}}$ atherectomy device in subjects affected with infrainguinal peripheral artery disease (PATHFINDER-I) [updated April 8, 2021]. https://clinicaltrials.gov/ct2/show/ NCT04229563?term $=$ NCT04229563\&draw $=2 \&$ rank=1.Zugegriffen: 25. Apr. 2021

49. Alperovich Z, Cohen O, Muncher Y, Ben-Oren I, Kuczmik W, Zelawski W et al (2021) Tissue postclassification using the measured acoustic signals during $355 \mathrm{~nm}$ laser atherectomy procedures. JBiophotonics 14(3):e202000185

50. Krievins DK, Halena G, Scheinert D, Savlovskis J, Szopinski P, Kramer A et al (2020) One-year results from the DETOUR I trial of the PQ Bypass DETOUR System for percutaneous femoropopliteal bypass. JVasc Surg 72(5):1648-1658e2

51. Schmidt A, Schreve MA, Huizing E, Del Giudice C, Branzan D, Unlu C et al (2020) Midterm outcomes of percutaneous deep venous arterialization with a dedicated system for patients with no-option chronic limb-threatening Ischemia: the ALPS multicenter study. JEndovasc Ther 27(4):658-665

52. Bates RJ, Toscano M, Balderman SC, Anagnostopoulos CE (1977) The cardiac veins and retrograde coronary venous perfusion. Ann Thorac Surg 23:83-90

53. Dotter CT, Judkins MP (1964) Transluminal treatment of arteriosclerotic obstruction. Description of a new technic and a preliminary report of its application. Circulation 30:654-670

54. Görtz H, Teßarek J, Berg P, Flessenkämper I, Ghotbi R, Hofmann M et al (2016) Novellierung des endovaskulären Curriculums zum 1.1.2017. Gefässchirurgie 21(6):440-446

55. Lawall H, Huppert P, Rümenapf G (2015) S3Leitlinie zur Diagnostik, Therapie und Nachsorge der peripheren arteriellen Verschlusskrankheit 